\title{
A case of Haemophilus parainfluenzae pneumonia
}

\author{
A Pillai, J L Mitchell, S L Hill, R A Stockley
}

\begin{abstract}
A 41 year old woman presented with community acquired pneumonia (CAP) which failed to resolve following treatment with amoxycillin and cefaclor prior to referral. Quantitative culture of sputum revealed a pure growth of Haemophilus parainfluenzae and, following antibiotic susceptibility testing of the isolate, ciprofloxacin was prescribed resulting in resolution of the infection. Immunological investigations showed that the patient had a high titre of $H$ parainfluenzae specific IgM. The combination of a pure growth of $H$ parainfluenzae, a response to appropriate antimicrobial therapy, and the presence of a specific antibody response indicated that this organism had a pathogenic role in the patient's pneumonia and should be considered in the differential diagnosis of CAP.

(Thorax 2000;55:623-624)
\end{abstract}

Keywords: Haemophilus parainfluenzae; pneumonia

Respiratory tract infections remain a common cause of morbidity and mortality worldwide and pneumonia in particular is the leading cause of death due to infectious diseases in developed countries. The microbiological aetiology of community acquired pneumonia (CAP) has changed over the last 20-30 years and, although Streptococcus pneumoniae is still the most prevalent pathogen, recent studies indicate an increasing incidence of pathogens such as Legionella spp, Mycoplasma spp, Chlamydia pneumoniae, Haemophilus influenzae, Moraxella catarrhalis, and viruses. ${ }^{1}$ In as many as $33 \%$ of cases, however, the microbial aetiology of the disease remains unidentified. ${ }^{2}$ Haemophilus parainfluenzae has been implicated in pneumonia but its role remains uncertain in view of its assumed low pathogenicity. ${ }^{3}$ We report details of a patient with pneumonia in whom an immune response was demonstrated to an isolate of $H$ parainfluenzae recovered as a pure growth from her sputum. The evidence indicates that this was the causative organism.

Respiratory Medicine,

Queen Elizabeth

Hospital, Edgbaston,

Birmingham B15 2TH,

UK

A Pillai

J L Mitchell

$S$ L Hill

R A Stockley

Correspondence to:

Professor R A Stockley

Received 15 April 1999

Returned to authors

9 June 1999

Revised manuscript received

27 September 1999

Accepted for publication

14 October 1999 her referral to the respiratory department in secondary care she developed some chest discomfort and was started on a slow release preparation of cefaclor.
When seen she looked reasonably well and clinical examination was normal apart from some fine middle lobe crackles. Her full blood count and biochemical profile were normal but the erythrocyte sedimentation rate (ESR) and $\mathrm{C}$ reactive protein level were raised $(105 \mathrm{~mm} / \mathrm{h}$ and $102 \mathrm{mg} / \mathrm{l}$, respectively). The chest radiograph showed consolidation of the middle lobe and, in view of these findings, she was commenced on clarithromycin $500 \mathrm{mg}$ twice a day and sputum was collected for culture.

Quantitative sputum culture ${ }^{4}$ revealed a pure growth of $H$ parainfluenzae at a concentration of $2.2 \times 10^{8}$ colony forming units $/ \mathrm{ml}$. No evidence of Streptococcus pneumoniae colonies was seen using this sensitive technique which involves the culture of serial dilutions of the homogenised sputum sample on blood and chocolate agar for 48 hours. The $H$ parainfluenzae isolate was fully susceptible to amoxycillin, augmentin, trimethoprim, cefuroxime, and ciprofloxacin, but resistant to erythromycin and clarithromycin. Treatment was therefore changed to ciprofloxacin $750 \mathrm{mg}$ twice daily. One week later she was much improved, producing only small amounts of clear sputum from which no recognised respiratory pathogens were recovered. Repeat ESR was $96 \mathrm{~mm} / \mathrm{h}$ but the $\mathrm{C}$ reactive protein level had fallen to $6 \mathrm{mg} / \mathrm{l}$. Two weeks later antibiotic treatment was stopped when ESR and C reactive protein had fallen to $37 \mathrm{~mm} / \mathrm{h}$ and $1 \mathrm{mg} / \mathrm{l}$, respectively.

A serum sample obtained before starting treatment with ciprofloxacin was tested for the presence of $\operatorname{IgG}, \operatorname{IgA}$ and $\operatorname{IgM}$ antibodies to $H$ parainfluenzae by an ELISA in which the capture antigen was prepared "in house" according to the method of Suzuki et al. ${ }^{5}$ In brief, the capture antigen consisted of eight distinct isolates of $H$ parainfluenzae (distinguished by differences in outer membrane protein profiles on SDS-PAGE) that were grown overnight in broth, harvested by centrifugation, and washed by resuspension in phosphate buffered saline (PBS, $\mathrm{pH}$ 7.4) followed by centrifugation as before. Isolates were resuspended in PBS, combined, sonicated (Soniprobe, Lucas Dawe Ultrasonics, UK), and centrifuged as before to remove whole cells. Total protein content was measured using a modified Lowry method and the sonicate was stored frozen in aliquots. Serum $H$ parainfluenzae specific IgG and IgA levels were 0.41 and 0.40 absorbance units respectively, which were within the ranges measured in nine healthy control subjects (IgG mean (SD) absorbance units $0.26(0.15)$, range $0.12-0.58$; IgA mean (SD) absorbance units 0.15 (0.11), range $0.02-0.35)$. The $\operatorname{IgM}$ titre of 0.65 , however, was raised compared with the range in healthy 


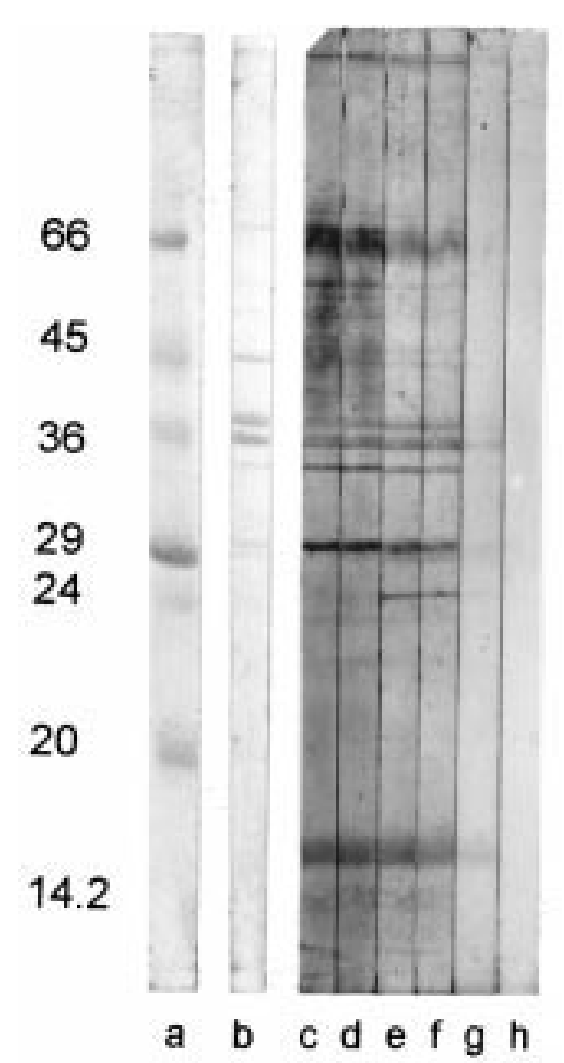

Figure 1 Outer membrane proteins of H parainfluenzae isolated from the sputum of a patient with pneumonia. Proteins were separated on a $12.5 \%$ SDS-PAGE gel, transferred to nitrocellulose, and immunoassayed for the presence of specific IgM in serum from the patient. Lane a $=$ low molecular weight markers; lane $b=$ total protein stain; lanes $c-g=$ serum diluted $1 / 5,1 / 10,1 / 50,1 / 100$ and $1 / 500$, respectively; lane $h=$ no serum control.

controls (mean (SD) 0.08 (0.07), range $0-0.24)$.

A western blot assay was used to confirm that outer membrane proteins prepared according to the method of Murphy and Apicella ${ }^{6}$ from the isolate of $H$ parainfluenzae recovered from the subject were recognised by specific IgM in the patient's serum up to a dilution of $1 / 500$ (fig 1). Serological testing by complement fixation revealed no other evidence of infection by $M$ pneumoniae, Chlamydia spp, Coxiella burnetii, influenza virus A or B, respiratory syncytial virus, or adenovirus.

\section{Discussion}

Traditionally, $H$ parainfluenzae has not been regarded as a respiratory tract pathogen. It has previously been identified in pneumonia ${ }^{78}$ but it remains unclear whether it was a contaminant or the actual pathogen involved. There have, to our knowledge, been no serological studies which clarify the role of this organism in lung infection. In the case we present there was a pure growth in high numbers of $H$ parainfluenzae from the sputum $\left(>10^{8} \mathrm{cfu} / \mathrm{ml}\right)$, a specific $\operatorname{IgM}$ antibody response to $H$ parainfluenzae antigens, and a clinical response. The failure to respond to the initial course of amoxycillin prescribed by the GP may reflect the inadequate length of treatment (five days) in a patient with an invasive infection. Given the therapeutic failure of amoxycillin in this case, and the data showing that $H$ parainfluenzae isolates often display reduced susceptibility to amoxycillin, ${ }^{910}$ the use of a quinolone may be an appropriate second line of treatment in pneumonias from which $H$ parainfluenzae has been isolated.

It is possible that $H$ parainfluenzae could have been a secondary pathogen following the initial pneumonia due to another aetiological agent; however, by the time the patient was referred to secondary care, only $H$ parainfluen$z a e$ was recovered despite extensive quantitative culture in a research laboratory specialising in respiratory infection. There was no evidence, based on serological testing, of either a viral or atypical aetiological agent. Nevertheless, whatever the sequence of events, the immune response in this patient indicates that $H$ parainfluenzae had played a pathogenic role in the illness.

This case suggests that $H$ parainfluenzae can cause pneumonia with systemic features in the community, even in apparently immunocompetent individuals. It should be considered as a possible pathogen, especially when conventional first line treatment fails.

1 Kayser FH. Changes in the spectrum of organisms causing respiratory tract infections: a review. Postgrad Med $\mathfrak{f}$ 1992;68(Suppl 3):S17-23.

2 Fang, GD, Fine M, Orloff J, et al. New and emerging etiologies for community-acquired pneumonia with implications or therapy: a prospective multicenter study of 359 cases. Medicine 1990;69:307-16.

3 Williams D, Perri M, Zervos MJ. Randomized comparative trial with ampicillin/sulbactam versus cefamandole in the therapy of community-acquired pneumonia. Eur $\mathcal{F}$ Clin Microbiol Infect Dis 1994;13:293-8.

4 Pye A, Stockley RA, Hill SL. Simple method for quantifying viable bacterial number in sputum. F Clin Pathol 1995;48: 719-24.

5 Suzuki S, Nakatomi Y, Sato H, et al. Haemophilus parainfluenzae antigen and antibody in renal biopsy samples and serum of patients with IgA nephropathy. Lancet 1994;343: $12-20$.

6 Murphy TF, Apicella MA. Antigenic heterogeneity of outer membrane proteins of nontypeable Haemophilus influenzae is a basis for a serotyping system. Infect Immunol 1985;50:15-21.

7 Poirier R. Comparative study of clarithromycin and roxithromycin in the treatment of community-acquired pneumonia. F Antimicrobial Chem 1991;27(Suppl A):10916.

8 Drehobl M, Bianchi P, Keyserling $\mathrm{CH}$, et al. Comparison of cefdinir and cefaclor in treatment of community-acquired pneumonia Antimicrob Agents Chemother 1997;41:1579-83.

9 Felmingham D, Gruneberg RN, and The Alexander Project Group. A multicentre collaborative study of the antimicrobial susceptibility of community-aquired, lower respiratory tract pathogens 1992-1993: the Alexander Project. $\mathcal{F}$ Antimicrob Chemother 1996;38(Suppl A):1-57.

10 Munday CJ, Johnson MM, Pye A, et al. Haemophilus parainfluenzae infection in chronic obstructive pulmonary disease. Chest 1997;112(Suppl):81S . 